\title{
Silibinin can enhance the osseointegration of titanium rods in ovariectomized rats maintenance treatment with valproic acid
}

zhoushan tao ( $\sim$ tzs19900327@126.com )

\section{Research Article}

Keywords: Osteoporosis, Osseointegration, Titanium implants, Silibinin, valproic acid

Posted Date: August 18th, 2021

DOl: https://doi.org/10.21203/rs.3.rs-618566/v2

License: (a) (i) This work is licensed under a Creative Commons Attribution 4.0 International License.

Read Full License 


\section{Abstract}

The present work was aimed to evaluate the effect of valproic acid(VPA) $₫$ Silibinin (SIM)+VPA on Ti rods osseointegration in ovariectomized rats and further investigation of the possible mechanism. The MC3T3-E1 cells were co-cultured with VPA囚SIM +VPA and induced to osteogenesis, and the cell viability $\mathbb{Z}$ mineralization ability were observed by MTT and ALP staining $₫$ Alizarin Red staining and Western blotting. Twelve weeks after bilateral ovariectomy, all animals were randomly divided into four groups:

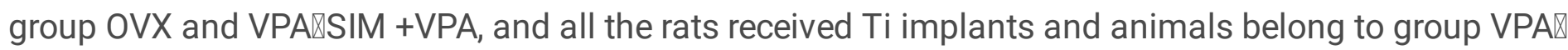
SIM +VPA received valproic acid(300 mg/ $\mathrm{kg} /$ day), valproic acid( $300 \mathrm{mg} / \mathrm{kg} /$ day) plus SIM $(25 \mathrm{mg} / \mathrm{kg} /$ day $)$, respectively, treatment until death at 12 weeks. Micro-CT, histology, biomechanical testing, bone metabolism index and Reverse transcription-quantitative polymerase chain reaction (RTqPCR) analysis were used to observe the therapeutic effect and explore the possible mechanism. Results shown that VPA decreased new bone formation around the surface of titanium rods and push-out force other than group OVX. Histology, Micro-CT and biochemical analysis results showed combined application of systemic VPA showed harmful effects than OVX group on bone formation in osteopenic rats, with the worse effects on CTX-1, P1NP and microarchitecture as well as biomechanical parameters by down-regulated gene expression of Runx2, OCN, Smad1, BMP-2 and OPG, while up-regulated RANKL. However, after SIM treatment, the above indicators were significantly improved. The present study suggests that systemic use of VPA may bring harm to the stability of titanium implants in osteoporosis, SIM can reverse the negative effect of VPA on the osseointegration of titanium rods in ovariectomized rats.

\section{Introduction}

With aging of worldwide population, osteoporosis, characterized by bone mass reduction and increased fractures, has become a global health problem that seriously affects the health of people, especially in middle-aged and aged women, which burdens the government financial and healthcare system greatly[1]. Owing to the micromotion between implant in osteoporotic bones, a higher rate of implant fixation failure has been discovered when compared with skeleton with normal bone mineral density[2]. However, the most important factor for success of orthopedic implants is the presence of sufficient bone at the implant site[3]. Metabolic disorders have a major impact on bone structure and may affect the processes involved in fracture healing as well as osseointegration $[4,5]$.

Seizure-related falls of epilepsy has been linked to several health outcomes, including osteopenia, osteoporosis, osteomalacia, falls and fractures, chronic pain and disability, and have up to six times increased fracture risk[6, 7]. Long-term treatment with antiepileptic drugs is accompanied by reduced bone mass that is associated with an increased risk of osteoporosis and fractures in patients with epilepsy[8]. Indeed, recent study have confirmed a negative role for a histone deacetylase inhibitor, valproic acid(VPA) on BMD of trochanter and Ward's triangle, when administered for 24 
months[9]. Potential mechanisms for bone loss after antiepileptic drugs treatment include vitamin D insufficiency, hyperparathyroidism, calcitonin deficiency, and inhibition of intestinal calcium absorption, and other hormonal changes[10, 11]. Silibinin (SIM) is specific inhibitor of 3-Hydroxy-3-methylglutarylcoenzyme A (HMG-CoA) that is widely used to reduce plasma cholesterol levels and prevent cardiovascular diseases[12]. In addition, it has been shown that SIM could affect bone turnover by inhibiting osteoclast activity through decreasing osteoclast formation, differentiation, proliferation, and function, and stimulating bone formation through reducing osteoblast apoptosis, and stimulating osteoblast formation[13]. In recent years, because of improvement the differentiation of bone marrow mesenchymal stem cells into osteoblasts and strengthening the function of osteoblasts, SIM has been paid more attention by scholars in the field of bone repair[14].

Despite this, there is limited evidence regarding the effects of SIM administration on osseointegration of implants for long-term maintenance therapy with VPA. We hypothesize that SIM could enhance titanium rods osseointegration in VPA-treated OVX rats. Therefore, the purpose of this study was to observe whether administration of SIM can reverse the efficacy of VPA on inhibition of osseointegration in osteoporotic bone.

\section{Materials And Methods}

\section{Experimental animals}

Health adult female Sprague-Dawley rats $(n=40)$, weighing $(200-250 \mathrm{~g}$, Central Laboratory of Yijishan Hospital) were used in this study. All rats were maintained in a temperature-controlled environment $\left(22 \pm 3^{\circ} \mathrm{C}\right), 55 \pm 5 \%$ relative humidity under a 12 -h light/dark cycle and humidity and fed standard laboratory food and water. Animal care and all experimental procedures were carried out in accordance with guidelines under the Animal Experimentation of Wannan Medical College.

\section{Cell culture, treatment and evaluation}

MC3T3-E1 cells, an osteoblast precursor cell line derived from mouse, were purchased from the Institute of Biochemistry and Cell Biology, CAS (Shanghai, China). MC3T3-E1 cells were cultured at $37^{\circ} \mathrm{C}$ and $5 \%$ $\mathrm{CO}_{2}$ atmosphere in DMEM containing $10 \% \mathrm{FBS}$. Thereafter, $\beta$-glycerophosphate $(10 \mathrm{mM})$, L-ascorbic acid $(50 \mu \mathrm{g} / \mathrm{mL})$ and dexamethasone $(10 \mathrm{nM})$ were added into DMEM to induce osteogenic differentiation. After reaching confluence, the DMEM were added with $\operatorname{VPA}(10 \mu \mathrm{M})$, or $\operatorname{SIM}(10 \mu \mathrm{M})$ treatment or no drug treatment(Control group, Con).

\section{MTT detection}


After 3 day treatment, $0.02 \mathrm{ml}$ of MTT solution $(5 \mathrm{mg} / \mathrm{ml})$ was added to each well and the plates were incubated at $37^{\circ} \mathrm{C}$ for $4 \mathrm{~h}$ to estimate cell viability. After that, the DMSO was added to dissolve formazan crystals. The absorbance value was measured at $570 \mathrm{~nm}$ using a plate reader (Bio-Rad Inc, Hercules, CA, USA).

\section{Alizarin Red and ALP staining}

To detect mineralization (calcium deposits) and ALP activity, 2 or 3 weeks after induction cells were fixed with $4 \%$ paraformaldehyde, and stained with Alizarin Red S (2\%; Sigma- Aldrich) or ALP staining kit (Beyotime Institute of Biotechnology, Shanghai, China) and the samples were observed by inverted phase contrast microscopy (Eclipse TE2000-U; Nikon, Japan).

\section{Western blot analyses}

To detect the expression levels of osteogenesis-related proteins, we performed western blot analyses. After 7 days treatment, the treated cells were harvested and the RIPA lysis buffer and BCA protein Assay(Beyotime, Shanghai, China) was used to extract and determin the total proteins. SDSpolyacrylamide gel electrophoresis (10\%) was performed to separate the sample protein (20 $\mu \mathrm{g} / \mathrm{lane}$ ). Then, the proteins were transferred to a polyvinylidene fluoride membrane (Millipore, Billerica, MA, USA). After blocking with $5 \%$ bovine serum albumin (Solarbio, Beijing, China) at room temperature for $2 \mathrm{~h}$ in Tris-buffered saline and Tween 20 (TBST), the membranes were probed with the primary antibodies such as with antibodies against the following proteins: alkaline phosphatase (ALP) (1:1000, ab33923), bone morphogenetic protein 2(BMP-2) (1:1000, ab135434), runt-related transcription factor 2 (RUNX-2), osteocalcin (OC) (1:1000, ab139412)凶type I collagen (COL-1) (1:1000, ab78034) (all from Abcam, Cambridge, UK); and GAPDH (1:1000, TA-08; ZSGB-Bio,Beijing, China)and incubated at $4^{\circ} \mathrm{C}$ overnight. The appropriate secondary antibody was applied (1:2000; horseradish peroxidase anti-mouse and horseradish peroxidase anti-rabbit) at room temperature for 1 hour. Quantitative analysis of protein expression was accomplished by scanning autoradiogram and densitometry (Image J, NIH).

\section{Surgery and treatment}

A bilateral ovariectomized(OVX) or sham operation was performed using standard methods, the rats were allowed for 12 weeks to conduct an osteoporosis as previous reports described[15, 16]. The femurs of each five randomly selected OVX rats and the five sham-operated ones were harvested for bone mineral density (BMD) evaluation by micro computed tomography(Micro-CT) and hematoxylin-eosin(HE) staining to confirm the establishment of osteoporosis. Afterwards all OVX animals were randomly divided into three experimental groups: OVX group, VPA and PTH+VPA group. There were 10 rats in each 
groups. Afterwards, the titanium rods [1 $\mathrm{mm}$ external diameter and $20 \mathrm{~mm}$ length(Zhejiang Guangci Medical Appliance Co., Ltd., Ningbo, China) ] were inserted bilaterally in all animals. The rats in OVX group, VPA and SIM+VPA group were treated with $1 \mathrm{ml}$ of isotonic saline; valproic acid (VPA) 300 $\mathrm{mg} / \mathrm{kg} /$ day, valproic acid (VPA) $300 \mathrm{mg} / \mathrm{kg} /$ day plus a gavage of simvastatin $(25 \mathrm{mg} / \mathrm{kg} /$ day), respectively. All drugs were purchased from SIGMA. The doses of SVP and SIM were determined according to previous experiments where they showed protective effects in OVX rodent models[17, 18]凶At 12 weeks postoperatively, all of the rats were euthanized by the over-dose administration of anesthetic agents. Subsequently, femur with implants and blood were harvested and evaluated by biochemical analysis, histological, Micro-CT, and push-out tests and Real-time quantitative RT-PCR (RT-qPCR).

\section{Micro-CT scanning}

For microarchitecture, trabecular bone architecture of defected area was determined by Micro-CT (MicroCT $\mu \mathrm{m}$ 100, SCANCO Medical, Switzerland). The

The entire trabecular compartment around implant from slice $2 \mathrm{~mm}$ below the growth plate to distal 100 slices was defined of volume of interest ( $\mathrm{VOI}$ ) to analysis the bone Osseointegration. The bone volume per total volume (BV/TV); trabecular number (Tb.N), thickness (Tb.Th), and spacing (Tb.Sp), the mean connective density (Conn.D), and the mean trabecular separation (Tb.Sp) within VOI were measured as previously described $[19,20]$.

\section{Histological examination}

Following Micro-CT scanning, the femur specimens were dehydrated by alcohols and embedded in PMMA. The specimens were cut into 200- $\mu \mathrm{m}$ thick sections using a microtome(Leica Microsystems Ltd, Wetzlar, Germany) and were subsequently ground and polished to a thickness of $40-50 \mu \mathrm{m}$ as previously described[21, 22]. The femur specimens were stained in Von-Gieson for histopathological examination.

\section{Mechanical test}

The right femora were cut at the level of both the proximal end of the rod and the distal end of it. After that, the distal ends of the femora were resected $2 \mathrm{~mm}$ in length, and then, the distal ends of the implants were exposed $2 \mathrm{~mm}$ in length. Each femur was fixed on a metal base with resin. The shear strength at the bone-implant interface was measured using a universal testing machine (Instron 4302; Instron, Norwood, MA, USA) operated in stroke-control mode at a constant displacement of $0.5 \mathrm{~mm} / \mathrm{min}$. A vector of the push-out force was applied parallel to the long axis of the rod. The peak load was obtained before failure to measure the bone-implant shear strength[23, 24]. 


\section{Biochemical analysis}

At the end of the experiment, blood was collected from the animals fasted overnight. Serum was separated and the aliquots were employed for the estimation of collagen type 1 cross-linked Ctelopeptide(CTX-1), procollagen I N-terminal propeptide (PINP). Rat antibody specific ELISA kits for CTX and PINP were procured from R\&D System, UK. The intra-assay coefficient of variance (CV) of CTX-1 is $10 \%$ and inter-assay CV: $11 \%$. The intra-assay CV of PINP is $7.4 \%$ and inter-assay CV is $8.0 \%$.

\section{Reverse transcription-quantitative polymerase chain reaction(RT-qPCR) analysis}

Total RNA was prepared and isolated using Trizol reagent (Invitrogen Corp., Carlsbad, CA, USA) as previously described[24,22]. Briefly, tissue samples around implant were homogenized using a mortar and pestle with liquid nitrogen, using $5 \mathrm{ml}$ of TRIzol per 50-100 mg of tissue. Insoluble material was removed from the homogenate by centrifugation for $10 \mathrm{~min}$ at $10,000 \mathrm{rpm}$ at $4^{\circ} \mathrm{C}$. RNA was reverse transcribed into cDNA using Primer Script RT reagent kit (Takara, Dalian, China) following standard procedures of the manufacturer's instructions. The quantitative real-time PCR was performed using the Applied Biosystems 7500 Sequence Detection System (ABI, Vernon, CA, USA). The expressions of the Runt-related transcription factor 2 (Runx-2), OC, osteocalcin; bone morphogenetic protein 2(BMP-2), small mothers against deca-pentaplegic homologs-1 (Smad1)『osteoprotegerin (OPG), and receptor activator of nuclear factor kappa-B ligand (RANKL) were normalized to a housekeeping gene, GAPDH.

Table 1 Nucleotide sequences for real-time RT-PCR primers.

\begin{tabular}{|lll|}
\hline Genes & Forward $\left(5^{\prime}-3^{\prime}\right)$ & Reverse $\left(5^{\prime}-3^{\prime}\right)$ \\
Runx-2 & TCCCAGTATGAGAGTAGGTGTCC & GGCTCAGATAAGAGGGGTAAGAC \\
OC & GAGGGCAGTAAGGTGGTGAA & CCTAAACGGTGGTGCCATAG \\
Smad1 & GTGGAAACAGGGCGACGAAG & AGGGAGCGAGGAATGGTGAC \\
BMP-2 & ACGACGGTAAAGGACATC & ATGGTTGGTGGAGTTCAG \\
RANKL & AGCCGAGACTACGGCAAGTA & AGACCACCTGACCCAGTCC \\
OPG & ACACACCAACTGCAGCTCAC & TGTCCACCAGAACACTCAGC \\
GAPDH & ACATTGTTGCATCAACGAC & TACTCAGCACCAGCATCACC \\
\hline
\end{tabular}




\section{Statistical analysis}

Measurement data were described as mean \pm standard deviation (mean $\pm \mathrm{SD}$ ). Analysis was were performed with commercially available statistical software (SPSS 19.0; SPSS Inc., Chicago, IL, USA). A one-way analysis of variance (ANOVA) and post-hoc Tukey test were carried out for comparisons between different groups. For all analyses, statistical significance was defined as $\mathrm{P}<0.05$.

\section{Results}

\section{Animals}

Due to anesthetic accident and infection, 5 rats died following the establishment ovariectomy or bone implant model. The survived 35 rats were included from further analysis.

\section{Confirmation of osteoporosis model}

The results of histological and Micro-CT performed on the distal metaphysis of femur in Figure 1. Compared with Sham group, the quantity and quality of trabecular bone were significantly lower than that in OVX group. In quantitative analysis of BMD, BMC, BV/TV, Tb.N, Tb. Sp and Tb.Th, statistically, there were obviously significant difference between OVX and the Sham group(Pख0.05).

\section{Osteoblastic Viability}

The cell viability by MTT assessment of three groups were shown in Figure 2. After VPA treatment, the cell activity of VPA group was significantly lower than that of Con group(Pख0.05), but the cell activity of SIM+VPA group was significantly higher than that of VPA group(Pख0.05).

\section{Alizarin red and ALP staining}

Alizarin red and ALP staining with quantification of area in osteogenic differentiation of MC3T3-E1 cells, as shown in Figure 3. The Mineralized nodules(number per well), Mineralized area (\%)『ALP grey value and ALP activity of VPA group is significantly higher than Con group $(p<0.05)$, but the values of osteogenic differentiation of MC3T3-E1 cells of SIM+VPA group was significantly higher than that of VPA group $(\mathrm{P} \otimes 0.05)$.

\section{Osteogenic Protein Expression of MC3T3-E1 cells}

Protein expression in osteogenic differentiation of MC3T3-E1 cells with different meddle, as shown in Figure 4. The values of osteogenic protein expression such as ALP $\square B M P-2 \square R U N X-2 \square O C$ and COL-1 in VPA group is significantly higher than Con group $(p<0.05)$, but the values of ALP $\square B M P-2 \square R U N X-2 \square O C$ and COL1 in SIM+VPA group was significantly higher than that of VPA group(Pख0.05). 


\section{Micro-CT evaluation}

The 3D reconstruction of the $\mathrm{VOI}$ area clearly depicted the effects of different treatments on bone

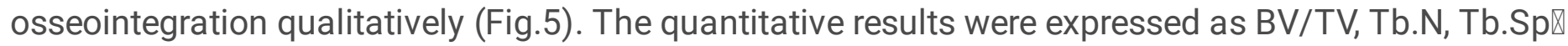
Conn.D and Tb.Th(Fig.6). Compared with OVX group, VPA group revealed the poor values of BV/TV, Tb.N, Tb.Sp $₫$ Conn.D and Tb.Th $(p<0.05)$. but the values of BV/TV, Conn.DQ Tb.N, Tb.Sp and Tb.Th in SIM+VPA group were significantly better than that of VPA group(P凶0.05).

\section{Histological analysis}

Histological images showing bone osseointegration for different treatment, as shown in Figure 7. Compared to group OVX, System administration VPA significantly decreased Bone area ratio(BAR) and Bone to implant contact $(\mathrm{BIC})$ in histomorphometric analysis $(\mathrm{P}<0.05$; Figure 8). However, SIM+VPA treatment revealed the highest values of BAR and BIC when compared with VPA group and OVX group $(p<0.05)$.

\section{Biomechanical test}

The maximum push-out force of different treatment were test as shown in Figure 9. Compared to group OVX, System administration VPA significantly decreased the maximum push-out force $(P<0.05$; Figure 9$)$. However, SIM +VPA treatment revealed the highest values of the maximum push-out force when compared with VPA group and OVX group $(p<0.05)$.

\section{RT-qPCR analysis}

Gene expression of bone tissue around implants after different treatment, as shown in Figure 10. At 12 weeks, VPA group showed decreased BMP-2, OC, RUNX-2, Smad1, OPG than the OVX group $(p<0.05)$, while VPA group exhibited increased RANKL than the OVX group $(p<0.05)$. However, SIM+VPA treatment presented the strongest effect on BMP-2, OC, RUNX-2, Smad1, OPG and RANKL when compared with VPA group and OVX group $(p<0.05)$.

\section{Bone turnover markers}

The bone turnover markers clearly depicted the differences between OVX and treated groups (Figure 11). Compared with group OVX, group VPA showed a higher CTX-1 and P1NP $(P<0.05)$. However, SIM+VPA treatment presented the stronger effect on CTX-1 and P1NP when compared with VPA group $(p<0.05)$.

\section{Discussion}

Nowadays, the treatment of osteoporotic fractures still a difficult point to clinicians, owing to the lower regeneration capacity of imbalanced bone remodeling as compared with the normal bone, as well as lack of Ti-implant specifically designed for osteoporotic bone even with a hydroxyapatite coating[25-27]. In 
recent years, as people pay attention to drugs that affect bone metabolism, more and more scholars have found that long-term use of antiepileptic drugs has negative effects on bone metabolism and bone mass[28-31]. In view of the possible effects of antiepileptic drugs on bone regeneration, this study selected clinically commonly used antiepileptic drug valproic acid, using an ovariectomized rat model to observe the effects of these drugs on Ti osseointegration; the results were verified our hypothesis that these drugs have a negative effect on osseointegration under osteoporosis. In addition, the bone formation promoting drug SIM was used to interfere with the ovariectomized rats treated with VPA, and the results confirmed the hypothesis that SIM could reverse the damage of osseointegration induced by VPA in osteoporosis.

In the current study, the osteogenic differentiation of MC3T3-E1 cells was evaluated, which were treatment with valproic acid. In vitro tests by MTT, ALP staining and Alizarin Red S staining indicated that the addition of valproic acid inhibit proliferation of MC3T3-E1 cells, as well as inducing MC3T3-E1 significant cell differentiation into osteoblasts. In vitro tests indicated that the addition of valproic acid constrain proliferation of MC3T3-E1 cells, and inhibit MC3T3-E1 significant cell differentiation into osteoblasts. MTT, ALP and RES assay revealed that valproic acid can effectively inhibit osteoblasts and reduce mineralization capacity. As we all know, differentiation and maturation of osteoblasts is a prerequisite to bone formation and is regulated by many cytokines such as ALP, BMP-2, COL-1, RUNX-2 and OC [32]. MC3T3-E1 osteoblasts have the potential to differentiate into osteocytes, and ALP, BMP-2, COL-1, RUNX-2 and OC is expressed at improper levels during MC3T3-E1 differentiation when cells was treated with valproic acid. However, when SIM, was added to the culture environment, there were significant changes in the above phenomena. The results of, MTT, ALP staining, Alizarin Red S staining and osteogenesis-related protein expression showed that the differentiation ability of MC3T3-E1 to osteoblasts was stronger in SIM +VPA group.

In vivo study exhibited that valproic acid treatment showed harmful effects in the context of OVX rat bone osseointegration. Compared with OVX group, Ti osseointegration treated with valproic acid showed a great variation in suppression tendency. Micro-CT images and histology showed that valproic acid treatment had lower new bone formation around implant than OVX group. Mechanical experiments further showed that valproic acid treatment had a detrimental effect on the stability of $\mathrm{Ti}$ in ovariectomized rats. In vivo and in vitro experiments indicate that clinically commonly used antiepileptic drug valproic acid have an inhibitory effect on bone formation around Ti. However, further treatment with SIM, Micro-CT and histological examination showed that the bone formation around Ti was significantly increased, and mechanical experiments showed that the stability of titanium rod was also significantly improved. 
To further investigate the regulatory role of VPA and SIM in Ti osseointegration, we detected the markers of bone metabolism and the expression of genes related to bone regulation. It is well known that BMP/SMAD signal pathway and OPG/RANKL signal pathway play an important role in bone remodeling. Bone marrow mesenchymal stem cells (BMSCs) are an expandable population of stem cells which can differentiate into osteoblasts. Bone marrow mesenchymal stem cells (BMSCs) differentiate to osteoblasts and form bones under the control of various osteogenic signal molecules and transcription factors[33]. In this process, BMP 2 plays an important role. BMP-2 can promote the differentiation of bone marrow mesenchymal stem cells into osteoblasts by activating SMAD1/5/8[34]. Runt-related transcription factor 2 (RUNX2) is a specific transcription factor of early osteoblasts and plays an important role in the formation of osteoblasts[1]. Subsequently, Osterix triggers osteoblasts to differentiate into osteoblasts through activating terminal osteogenic marker genes such as osteocalcin (OC)[35]. Receptor activator of NF-KB ligand (RANKL), which binds to its receptor (RANK) displayed by the osteoclast precursor to induce its maturation into the osteoclast, and osteoprotegerin (OPG) preventing it from interacting with the RANK recepto[36]. The relative levels of RANKL and OPG expression in the bone microenvironment are the key to determining osteoclast formation and activity.

In this study, with VPA treatment, the expression of Runx2, OC, Smad1, BMP-2 and OPG gene in bone tissue decreased significantly, while the level of RANKL increased significantly, which indicated that the OPG/RANKL and BMP-2/Smad1 signal pathway was inhibited, which led to the inhibition of osteoblast activity and the increase of osteoclast activity, and finally decreased the ability of bone repair and osseointegration. Among them, the increase of CTX-1 and P1NP, an index of bone metabolism, can further explain this performance. Furthermore, this finding was also supported by some previous investigations[28, 37]. However, further treatment with SIM, RT-PCR examination showed that the expression of Runx2, OC, Smad1, BMP-2 and OPG gene in bone tissue increased significantly, while the level of RANKL decreased significantly, which indicated that the OPG/RANKL and BMP-2/Smad1 signal pathway were activation, and finally enhance the ability of bone repair and osseointegration.

There are several shortcomings in this study. First of all, only one antiepileptic drug was selected in this study, which does not mean that other antiepileptic drugs also have harmful effects on osseointegration. Secondly, owing to only one drug dose was selected for intervention for a period of 12 months in this study, it is difficult to determine whether this harmful effect is related to drug dose and treatment time. At the same time, this study only use one anti-osteoporosis drugs, and we will explore whether other antiosteoporosis drugs can reverse the negative effects of antiepileptic drugs on osseointegration in the future. 
In conclusion, the present study suggests that systemic use of VPA may bring harm to the stability of titanium implants in patients with osteoporosis, and this effect may be related to the inhibition of OPG/RANKL and BMP-2/Smad1 signal pathway. Our currently study further demonstrated that SIM can antagonize the negative effects of VPA on osteoblasts and osteoporotic rats, and improve the osseointegration ability of Ti in osteoporotic state.

\section{Declarations}

\section{Acknowledgments}

This study was supported by a grant from the natural science foundation for education department of Anhui Province (Grant No. KJ2017A266), natural science foundation of Anhui Province (Grant No: 1708085QH209) and national Natural Science Foundation of China (Grant No. 81772348).

\section{References}

1. Y L, H W, XZ Z, N L, YC G, TP C. Pentraxin 3 promotes the osteoblastic differentiation of MC3T3-E1 cells through the PI3K/Akt signaling pathway. Bioscience reports. 2020;40(6). doi:10.1042/bsr20201165.

2. Takahashi E, Kaneuji A, Tsuda R, Numata Y, Ichiseki T, Fukui K et al. The influence of cement thickness on stem subsidence and cement creep in a collarless polished tapered stem:When are thick cement mantles detrimental? Bone \& joint research. 2017;6(5):351-7.

3. Oki Y, Doi K, Makihara Y, Kobatake R, Kubo T, Tsuga K. Effects of continual intermittent administration of parathyroid hormone on implant stability in the presence of osteoporosis: an in vivo study using resonance frequency analysis in a rabbit model. Journal of Applied Oral Science. 2017;25(5):498-505.

4. Nikolaou VS, Efstathopoulos N, Kontakis G, Kanakaris NK, Giannoudis PV. The influence of osteoporosis in femoral fracture healing time. Injury Extra. 2009;40(10):202.

5. Debby H, Hom-Lay W. Medical contraindications to implant therapy: Part II: Relative contraindications. Implant dentistry. 2007;16(1):13-23.

6. M F, ML W, BY W. Survey of risk factors for osteoporosis and osteoprotective behaviors among patients with epilepsy. Epilepsy \& behavior : E\&B. 2015;45:217-22. doi:10.1016/j.yebeh.2015.01.021.

7. N J, LM L, CJ M, HJ P, J M, WD L. Association of antiepileptic drugs with nontraumatic fractures: a population-based analysis. Archives of neurology. 2011;68(1):107-12. doi:10.1001/archneurol.2010.341.

8. PC S, DJ W, JG W, TP VS, AC E. Use of antiepileptic drugs and risk of fractures: case-control study among patients with epilepsy. Neurology. 2006;66(9):1318-24.

doi:10.1212/01.wnl.0000210503.89488.88. 
9. Ecevit C, Aydogan A, Kavakli T, Altinoz S. Effect of carbamazepine and valproate on bone mineral density. Pediatr Neurol. 2004;31(4):279-82. doi:10.1016/j.pediatrneurol.2004.03.021.

10. S K, P K, KK P, D V. Homocysteine in neurological disease: a marker or a cause? CNS \& neurological disorders drug targets. 2011;10(3):361-9. doi:10.2174/187152711794653797.

11. S K, KK P, D V. Insights into liaison between antiepileptic drugs and bone. Drug discovery today. 2009;14:428-35. doi:10.1016/j.drudis.2009.01.004.

12. Chen HH, Huang XH, Yao-Yao LI, Qi LI, Lan C, Hao MM. Simvastatin prevents alveolar bone loss in an experimental rat model of periodontitis after ovariectomy. Journal of translational medicine. 2014;12(1):284.

13. Ahmad O, Soodeh A, Ali M, Nicola M. Bone regenerative medicine: classic options, novel strategies, and future directions. Journal of Orthopaedic Surgery and Research,9,1(2014-03-17). 2014;9(1):18.

14. Weixi W, Nyman JS, Moss HE, Gloria G, Mundy GR, Xiangli Y et al. Local low-dose lovastatin delivery improves the bone-healing defect caused by Nf1 loss of function in osteoblasts. Journal of Bone \& Mineral Research. 2010;25(7):1658-67.

15. ZS T, WS Z, XJ W, X Z, L W, JB X et al. Prevention of ovariectomy-induced osteoporosis in rats : Comparative study of zoledronic acid, parathyroid hormone (1-34) and strontium ranelate. Zeitschrift fur Gerontologie und Geriatrie. 2019;52(2):139-47. doi:10.1007/s00391-018-1376-x.

16. ZS T, YX L, W C, ZL H, KK T, Q Z et al. Effect of teriparatide on repair of femoral metaphyseal defect in ovariectomized rats. Zeitschrift fur Gerontologie und Geriatrie. 2016;49(5):423-8. doi:10.1007/s00391015-0949-1.

17. Shahrezaee M, Oryan A, Bastami F, Hosseinpour S, Shahrezaee MH, Kamali A. Comparative impact of systemic delivery of atorvastatin, simvastatin, and lovastatin on bone mineral density of the ovariectomized rats. Endocrine. 2018;60(1):138-50. doi:10.1007/s12020-018-1531-6.

18. Parveen B, Tiwari AK, Jain M, Pal S, Chattopadhyay N, Tripathi M et al. The anti-epileptic drugs valproate, carbamazepine and levetiracetam cause bone loss and modulate Wnt inhibitors in normal and ovariectomised rats. Bone. 2018;113:57-67. doi:10.1016/j.bone.2018.05.011.

19. Li YF, Li XD, Bao CY, Chen QM, Zhang H, Hu J. Promotion of peri-implant bone healing by systemically administered parathyroid hormone (1-34) and zoledronic acid adsorbed onto the implant surface. Osteoporosis international : a journal established as result of cooperation between the European Foundation for Osteoporosis and the National Osteoporosis Foundation of the USA. 2013;24(3):1063-71. doi:10.1007/s00198-012-2258-5.

20. Gabet Y, Kohavi D, Kohler T, Baras M, Müller R, Bab I. Trabecular bone gradient in rat long bone metaphyses: mathematical modeling and application to morphometric measurements and correction of 
implant positioning. Journal of Bone and Mineral Research. 2008;23(1):48-57.

21. ZS T, XJ W, WS Z, XJ W, W L, M Y et al. Local administration of aspirin with $\beta$-tricalcium phosphate/poly-lactic-co-glycolic acid ( $\beta$-TCP/PLGA) could enhance osteoporotic bone regeneration. Journal of bone and mineral metabolism. 2019;37(6):1026-35. doi:10.1007/s00774-019-01008-w.

22. Z T, W Z, X W, H L, N M, Y L et al. Local administration of aspirin improves osseointegration of hydroxyapatite-coated titanium implants in ovariectomized rats through activation of the Notch signaling pathway. Journal of biomaterials applications. 2020;34(7):1009-18. doi:10.1177/0885328219889630.

23. Li Y, Li Q, Zhu S, Luo E, Li J, Feng G et al. The effect of strontium-substituted hydroxyapatite coating on implant fixation in ovariectomized rats. Biomaterials. 2010;31(34):9006-14.

24. ZS T, XJ W, M Y, HG X. Local administration with silymarin could increase osseointegration of hydroxyapatite-coated titanium implants in ovariectomized rats. Journal of biomaterials applications. 2019;34(5):664-72. doi:10.1177/0885328219863290.

25. Uezono M, Takakuda K, Kikuchi M, Suzuki S, Moriyama K. Hydroxyapatite/collagen nanocompositecoated titanium rod for achieving rapid osseointegration onto bone surface $\dagger$. Journal of Biomedical Materials Research Part B Applied Biomaterials. 2013;101B(6):1031-8.

26. N L-V, J F-F, JM R, BM S, S H-H, A L-V. Bioactive Surfaces vs. Conventional Surfaces in Titanium Dental Implants: A Comparative Systematic Review. Journal of clinical medicine. 2020;9(7). doi:10.3390/jcm9072047.

27. D X, Y W, Z L, C W, Q Z, C D et al. Tailorable hierarchical structures of biomimetic hydroxyapatite micro/nano particles promoting endocytosis and osteogenic differentiation of stem cells. Biomaterials science. 2020;8(12):3286-300. doi:10.1039/d0bm00443j.

28. B P, AK T, M J, S P, N C, M T et al. The anti-epileptic drugs valproate, carbamazepine and levetiracetam cause bone loss and modulate Wnt inhibitors in normal and ovariectomised rats. Bone. 2018;113:57-67. doi:10.1016/j.bone.2018.05.011.

29. A S, S M, E C, L P, G S, L G. Antiepileptic Drugs and Bone Health: Current Concepts. Psychopharmacology bulletin. 2020;50(2):36-44.

30. AA P, S A, M S, K J, C E, J H et al. Bone Health Screening Practices Among Neurologists in Patients on Antiepileptic Drugs: A Quality Improvement Project. Pediatric neurology. 2020;102:49-55. doi:10.1016/j.pediatrneurol.2019.06.020.

31. J F, L P, J L, T T, Y C. Effects of Second-Generation Antiepileptic Drugs Compared to First-Generation Antiepileptic Drugs on Bone Metabolism in Patients with Epilepsy: A Meta-Analysis. Hormone and metabolic research $=$ Hormon- und Stoffwechselforschung $=$ Hormones et metabolisme. 2019;51(8):51121. doi:10.1055/a-0963-0054. 
32. JC dSdO, ER L, CK S, R O, IR G-J. Immunohistochemistry evaluation of BMP-2 with $\beta$-tricalcium phosphate matrix, polylactic and polyglycolic acid gel, and calcium phosphate cement in rats. Oral and maxillofacial surgery. 2017;21(2):247-58. doi:10.1007/s10006-017-0624-3.

33. D H, X H, D Z, Q Z, C Y. Two novel polysaccharides from rhizomes of Cibotium barometz promote bone formation via activating the BMP2/SMAD1 signaling pathway in MC3T3-E1 cells. Carbohydrate polymers. 2020;231:115732. doi:10.1016/j.carbpol.2019.115732.

34. H L, B N, Z D, S Z, T L, B Y. Bacitracin promotes osteogenic differentiation of human bone marrow mesenchymal stem cells by stimulating the bone morphogenetic protein-2/Smad axis. Biomedicine \& pharmacotherapy = Biomedecine \& pharmacotherapie. 2018;103:588-97.

doi:10.1016/j.biopha.2018.04.084.

35. CY T, W C, Y L, J W, Y Z, A M et al. Runx1 up-regulates chondrocyte to osteoblast lineage commitment and promotes bone formation by enhancing both chondrogenesis and osteogenesis. The Biochemical journal. 2020;477(13):2421-38. doi:10.1042/bcj20200036.

36. YW G, K F, XL L, HF C, ZY S, CF W et al. The Recombinant Protein EphB4-Fc Changes the Ti ParticleMediated Imbalance of OPG/RANKL via EphrinB2/EphB4 Signaling Pathway and Inhibits the Release of Proinflammatory Factors In Vivo. Oxidative medicine and cellular longevity. 2020;2020:1404915. doi:10.1155/2020/1404915.

37. SS D, AS S, P E, M E, M D, NB A et al. Effects of carbamazepine, eslicarbazepine, valproic acid and levetiracetam on bone microarchitecture in rats. Pharmacological reports : PR. 2020. doi:10.1007/s43440-020-00087-1.

\section{Figures}



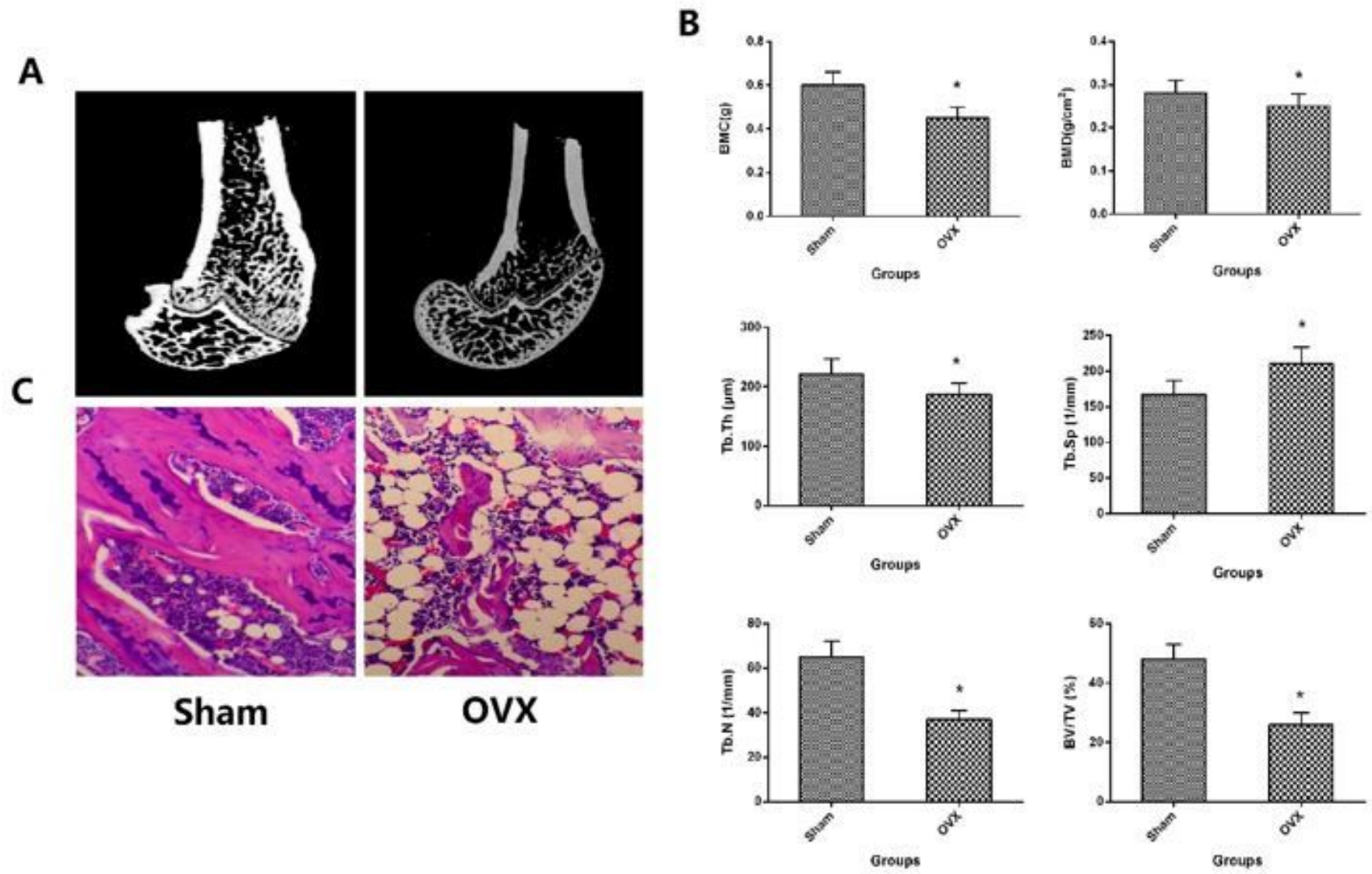

\section{Figure 1}

The Micro-CT 3D scanning of trabecular bone at femoral metaphyseal and quantitative results expressed as BMD, BMC, BV/TV, Tb.N, Tb.Sp and Tb.Th(A,B), as well as histological analysis(C) of femoral metaphysis. ${ }^{*} p<0.05 \mathrm{vs}$. Sham group, the scale bar represents $1 \mathrm{~mm}$. 


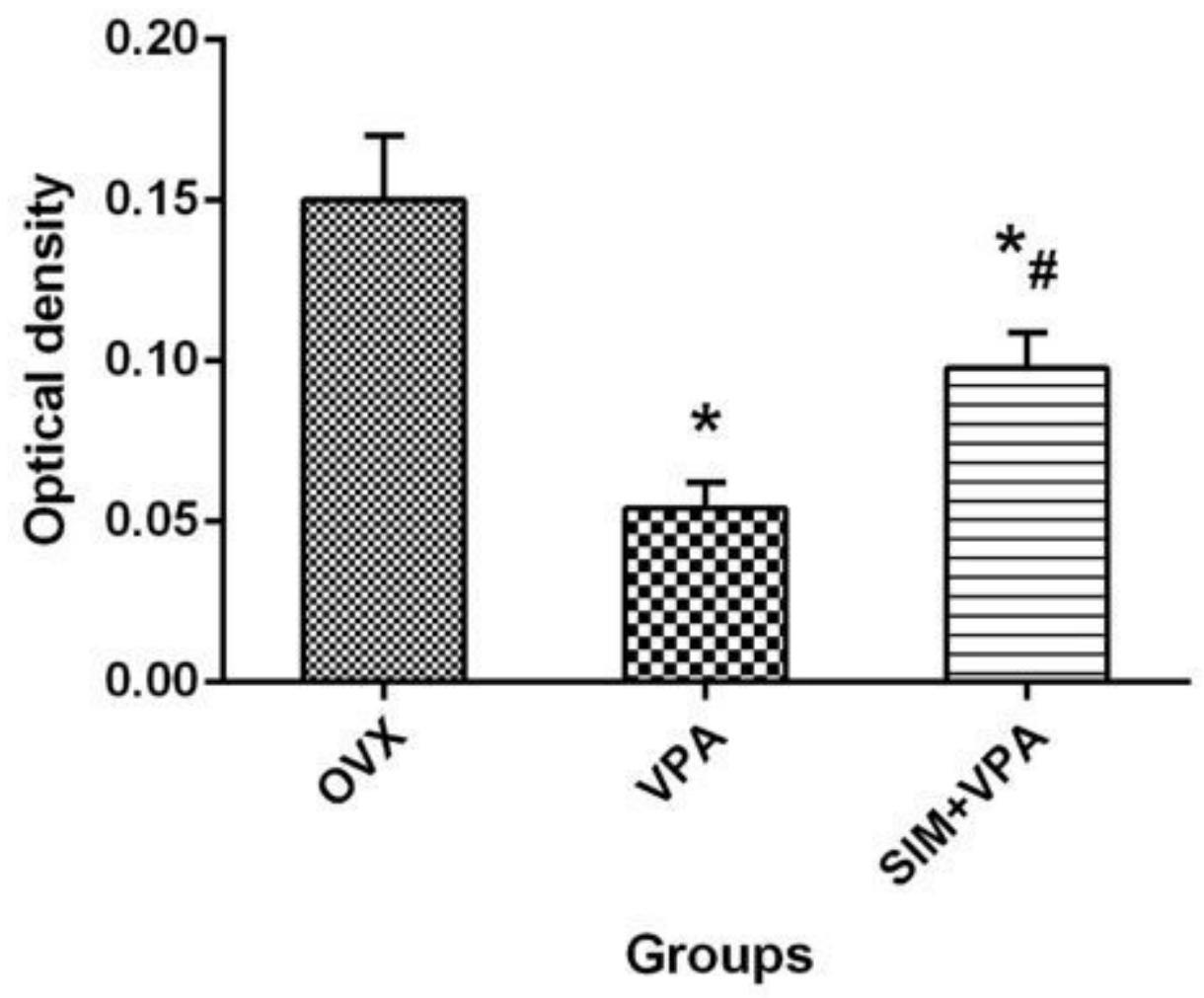

Figure 2

MTT assay of cell viability with different treatment. ${ }^{*} p<0.05$ vs. Con group, $\# p<0.05$ vs. VPA group. 

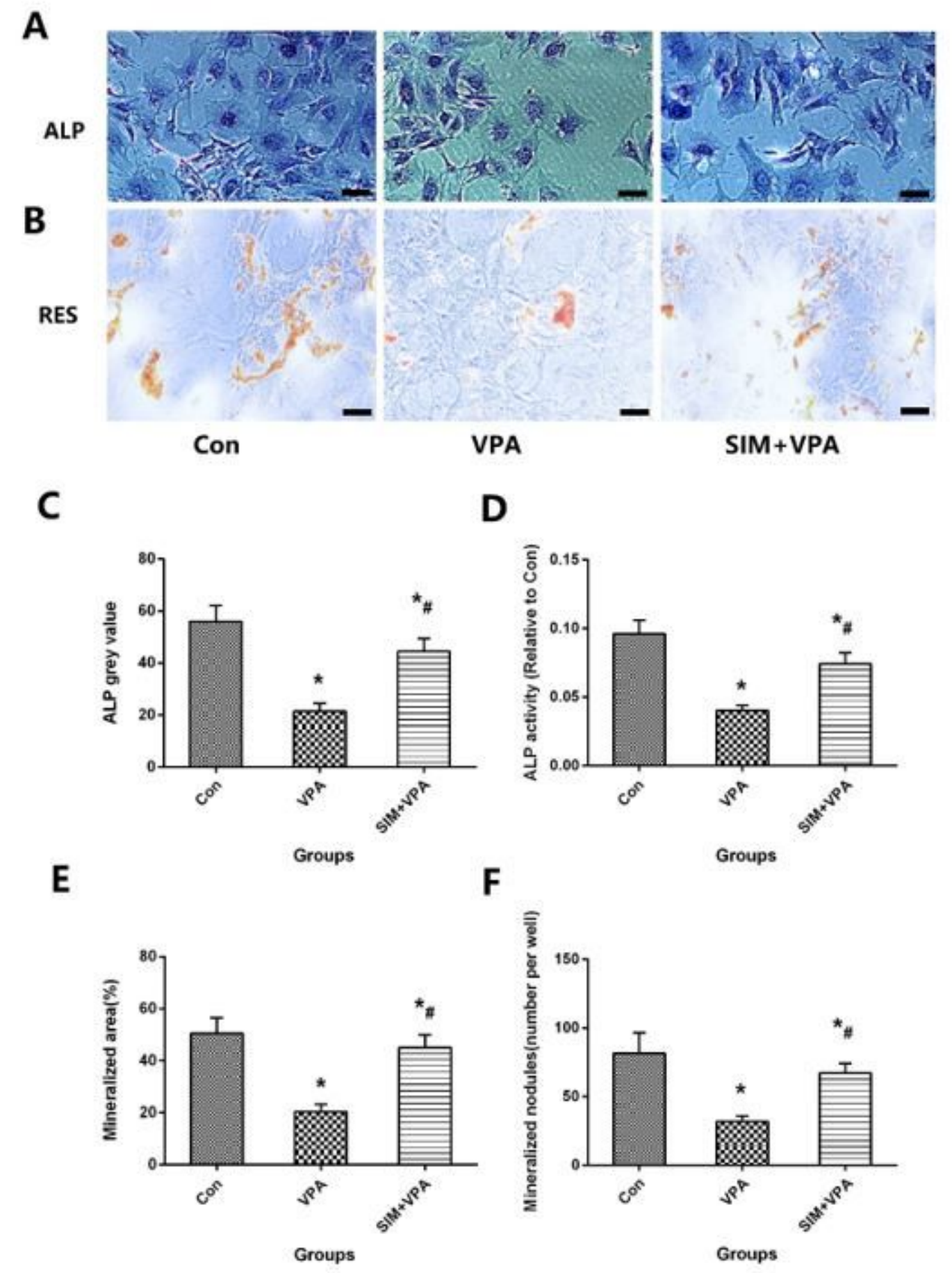

\section{Figure 3}

Representative images of alizarin red staining and ALP staining, ALP grey value of staining, ALP activity, Mineralized nodules(number per well) and Mineralized area (\%)of different groups. ${ }^{\star} p<0.05$ vs. Con group, $\#$ p<0.05 vs. VPA group. The scale bar represents $100 \mu \mathrm{m}$. 

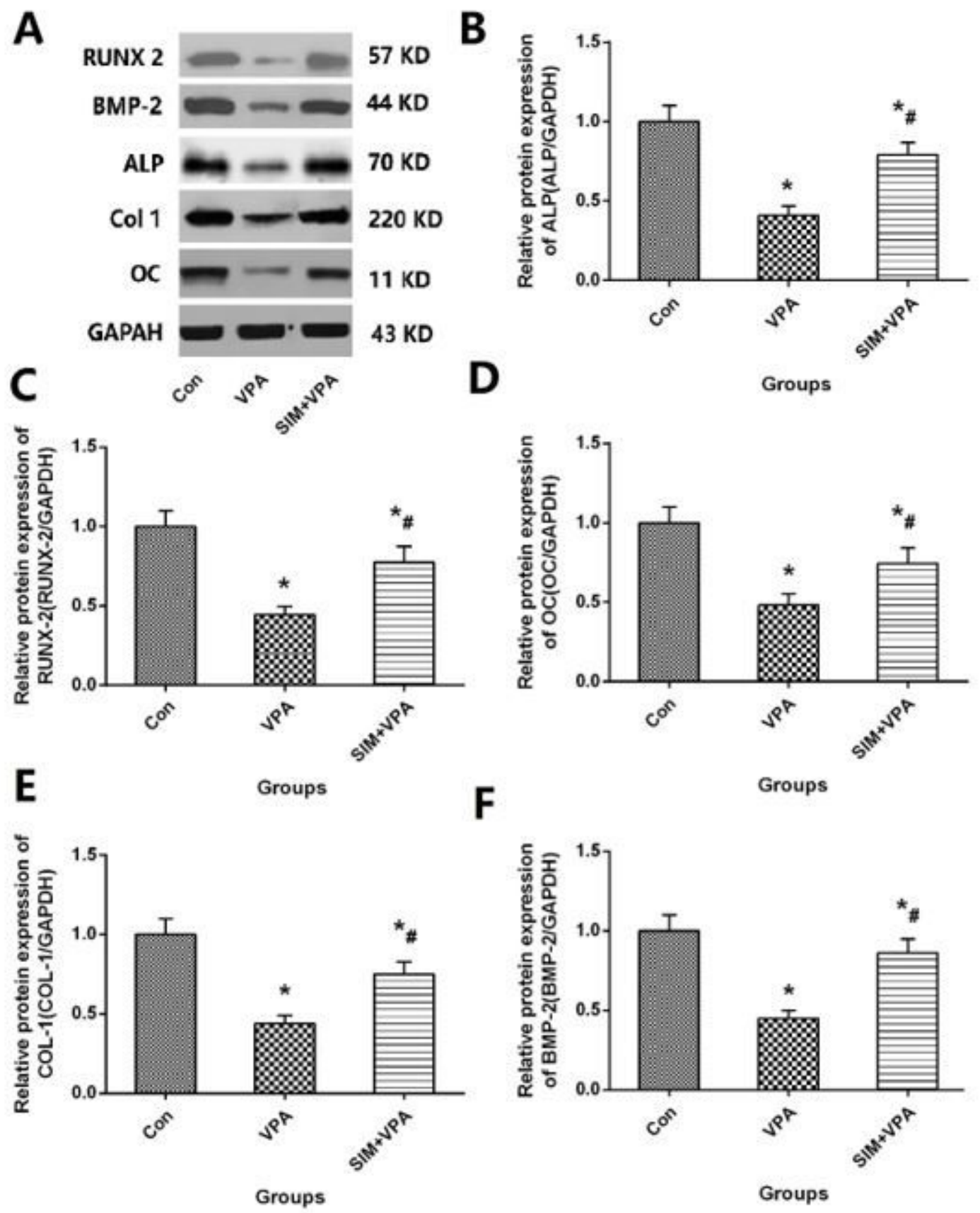

$\mathbf{F}$

Groups

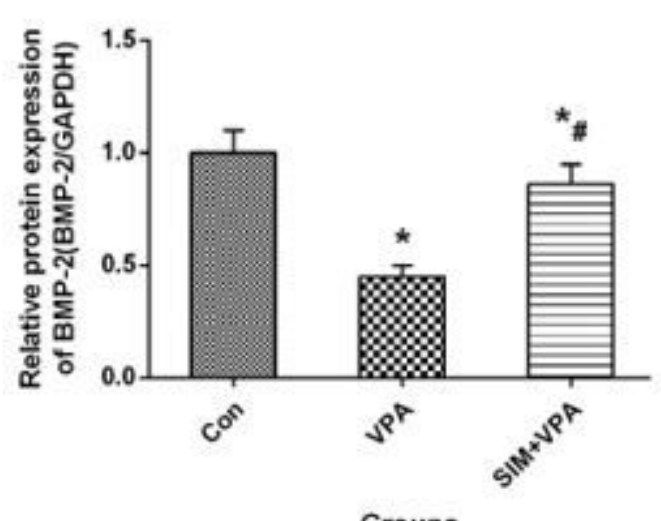

Groups

Figure 4

A Representative images of Osteogenic Protein Expression and Osteogenic Protein Expression for BMP-2ם ALP $\square R U N X-2 \square O C$ and COL-1(A-F) of MC3T3-E1 cells; * $p<0.05$ vs. Con group, $\# p<0.05$ vs.VPA group. 

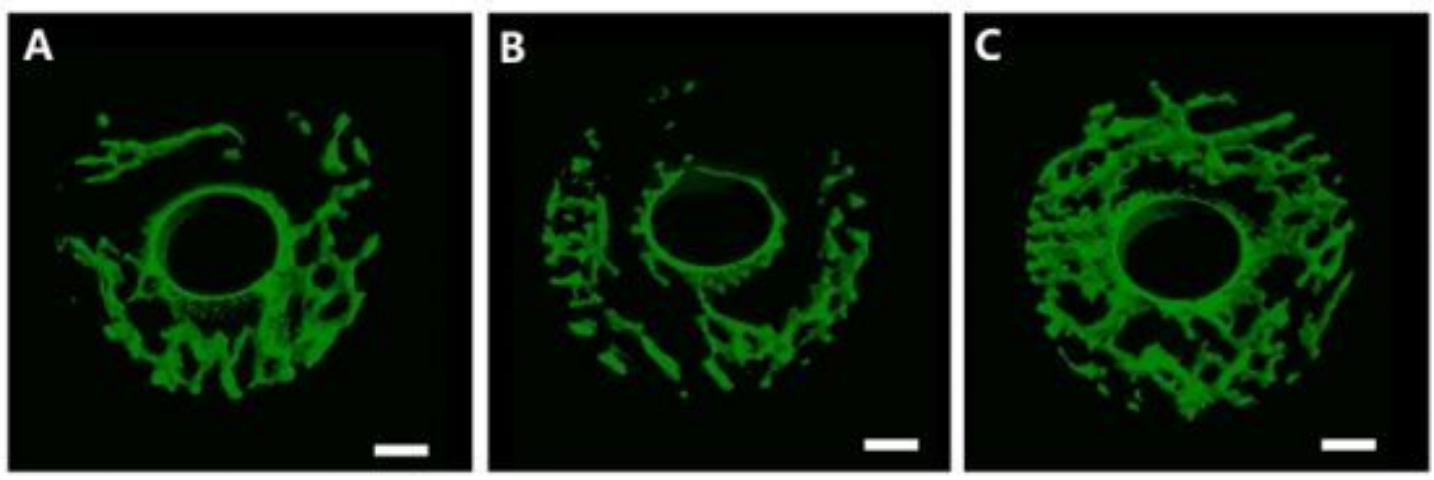

Figure 5

Representative images of the 3D reconstruction of Bone osseointegration around implants. The scale bar represents $1 \mathrm{~mm}$.
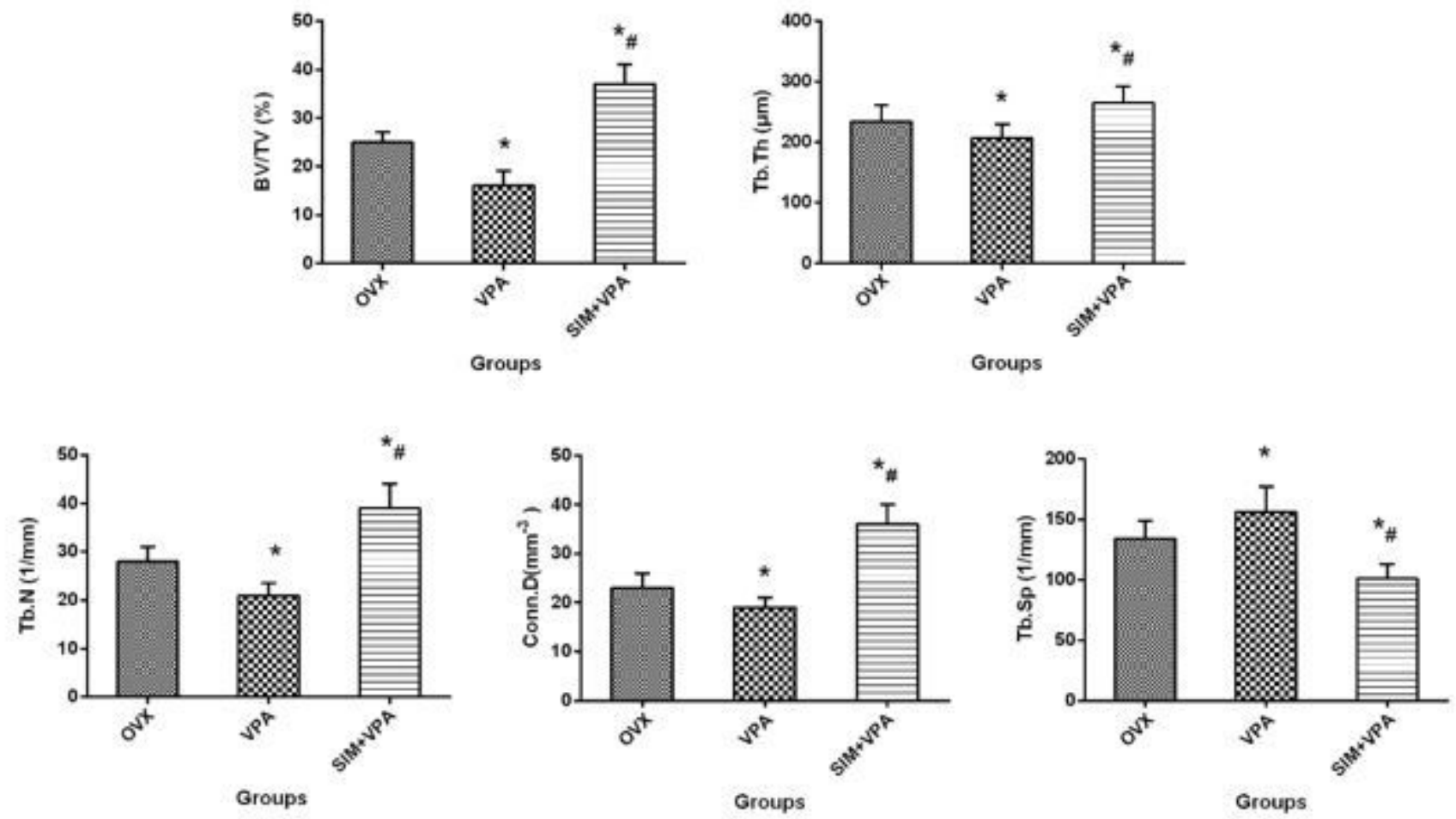

Figure 6

Quantitative results expressed as BV/TV, Tb.N, Tb.Sp $₫$ Conn.D and Tb.Th of VOI area. ${ }^{*} p<0.05$ vs. Con group, \# $\mathrm{p}<0.05$ vs. VPA group. 

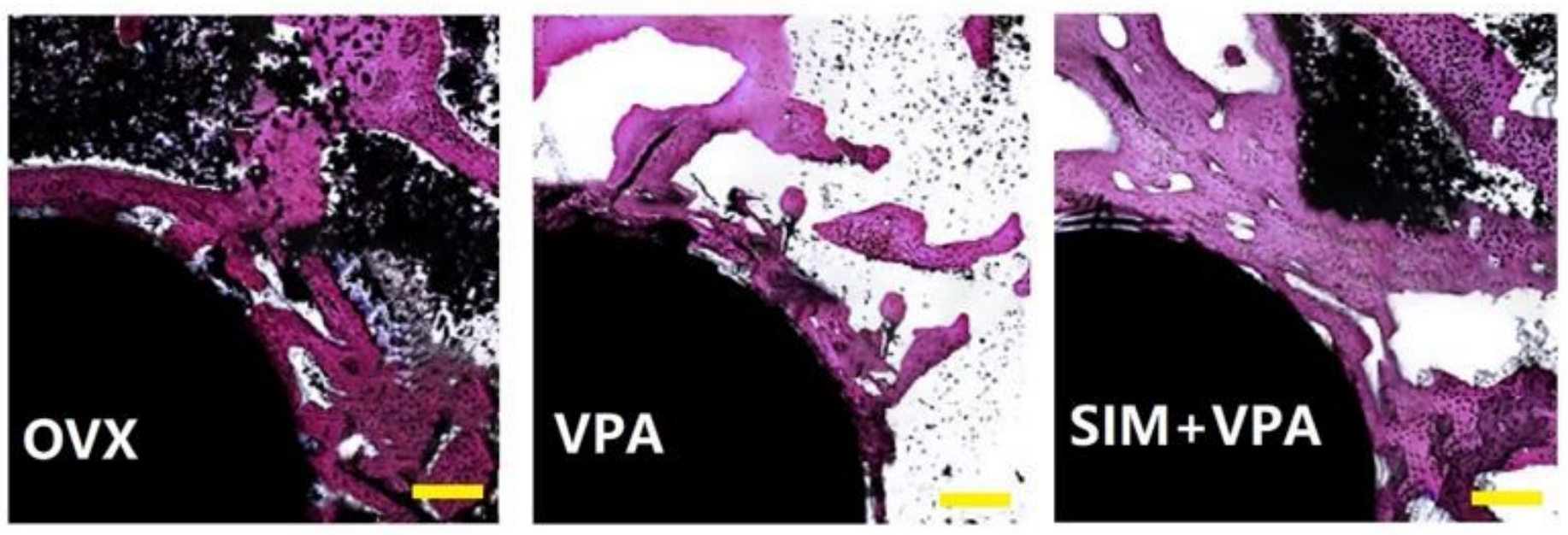

Figure 7

Histological analysis through toluidine blue Von-Gieson (scale bar= $200 \mu \mathrm{m}$ ).

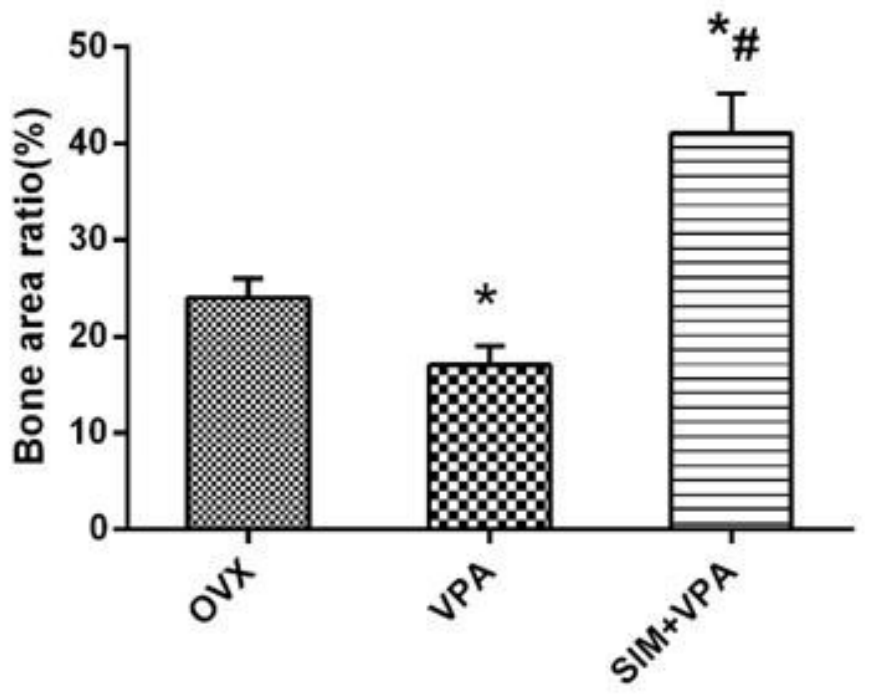

Groups

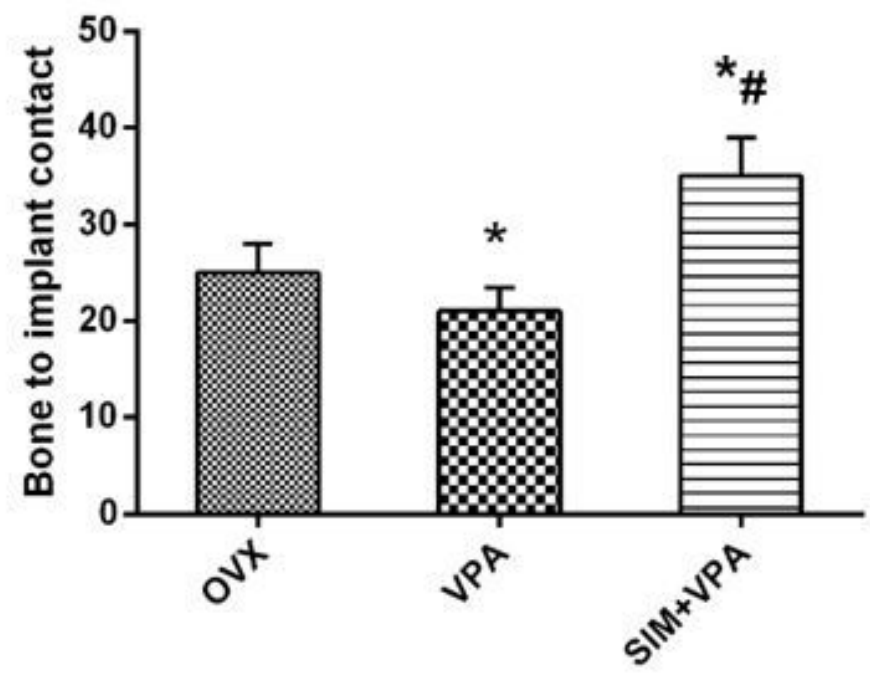

Groups

Figure 8

BIC and BAR of implants after 12 weeks treatment. ${ }^{*} p<0.05$ vs. Con group, $\# p<0.05$ vs. VPA group. 


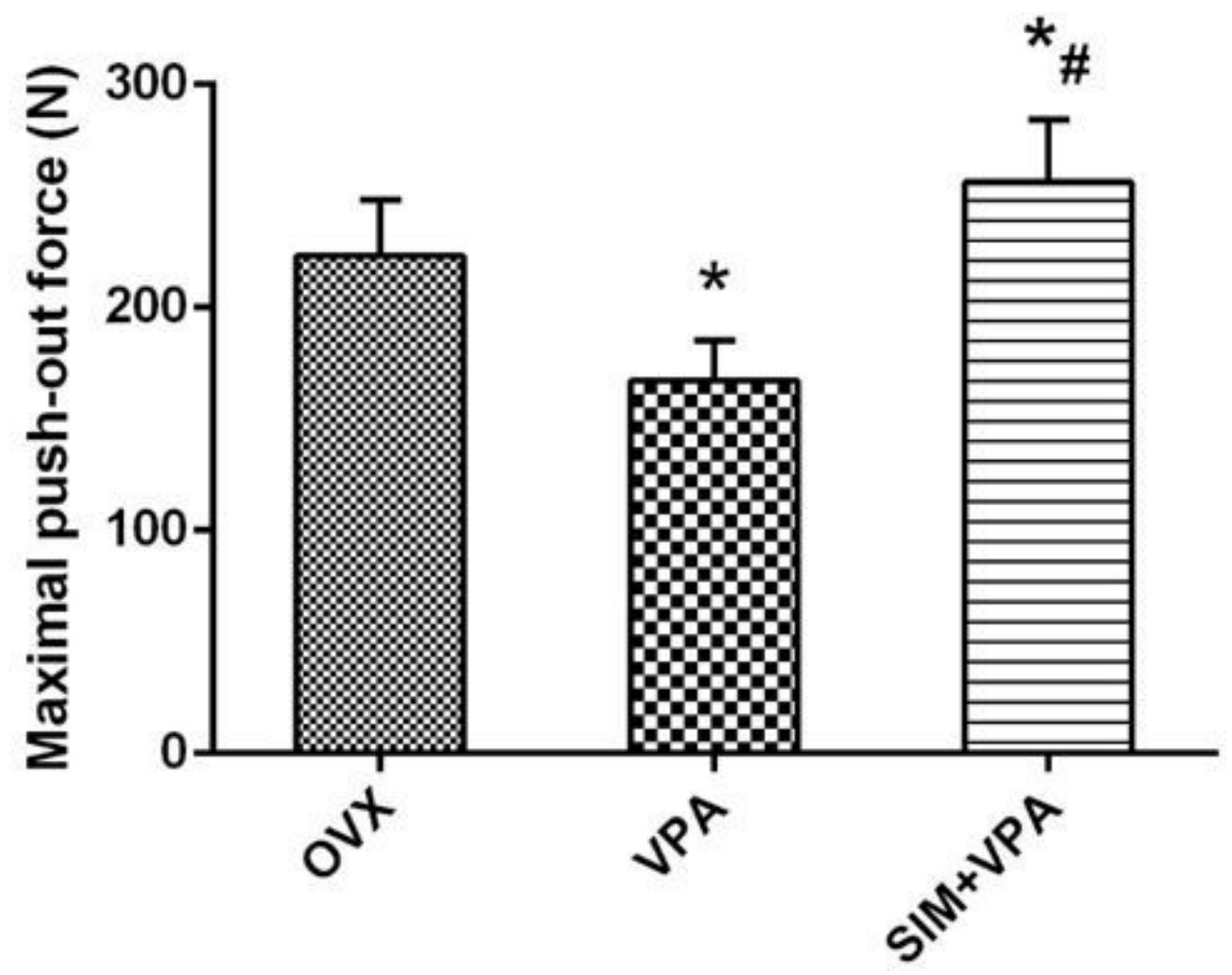

Groups

Figure 9

The maximum push-out force of different treatment, ${ }^{*} p<0.05$ vs. Con group, $\# p<0.05$ vs. VPA group. 


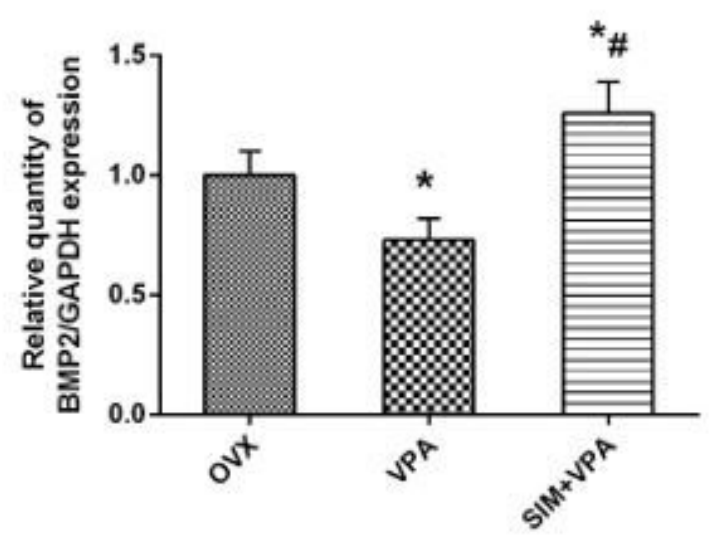

Groups

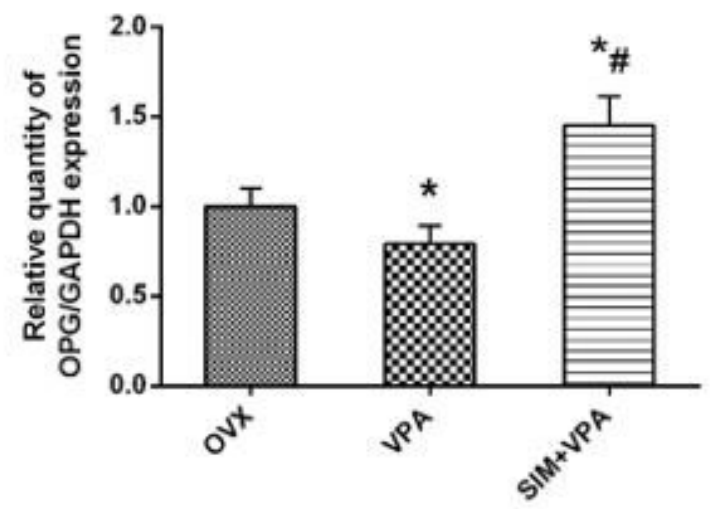

Groups

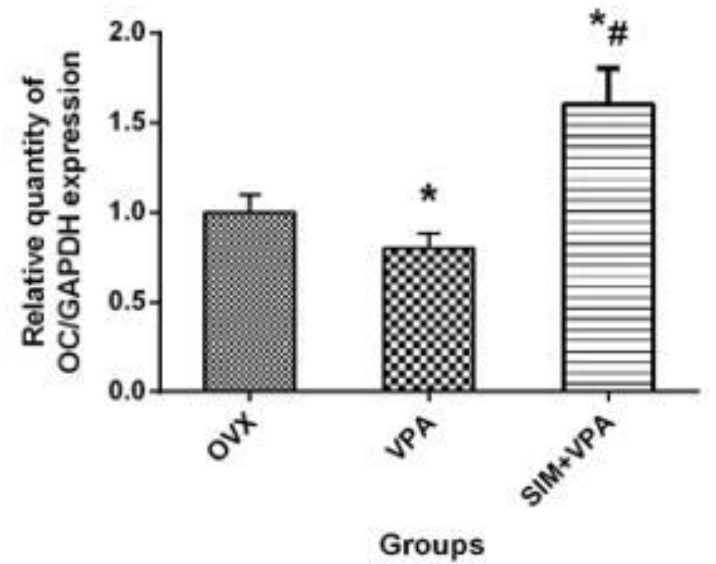

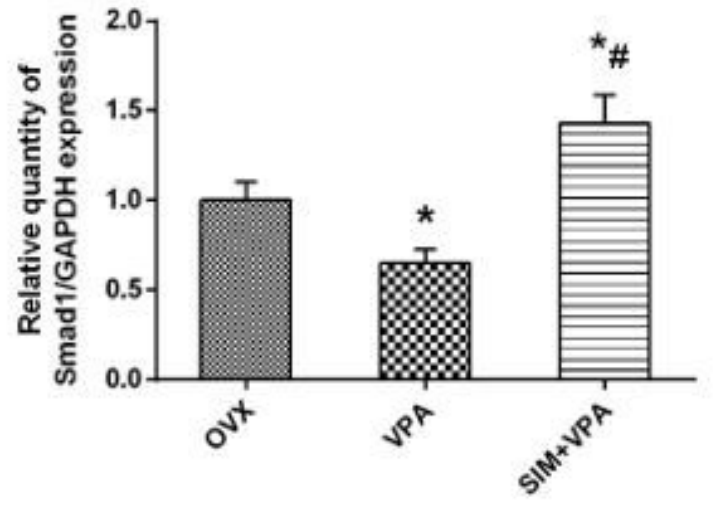

Groups

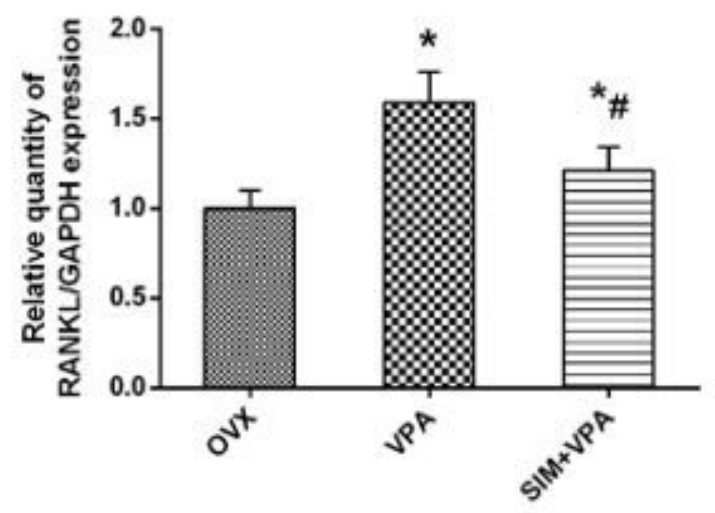

Groups

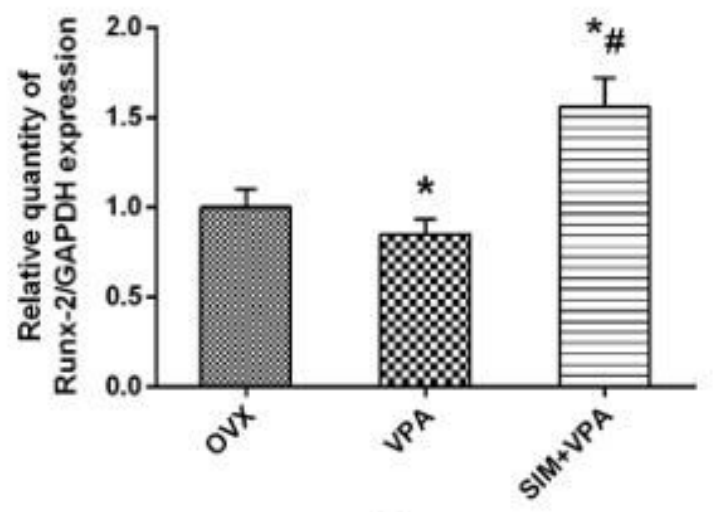

Groups

Figure 10

Gene expression of VIO area bone tissue after different treatment. ${ }^{*}<<0.05$ vs. OVX group, $\# p<0.05$ vs. VPA group. 

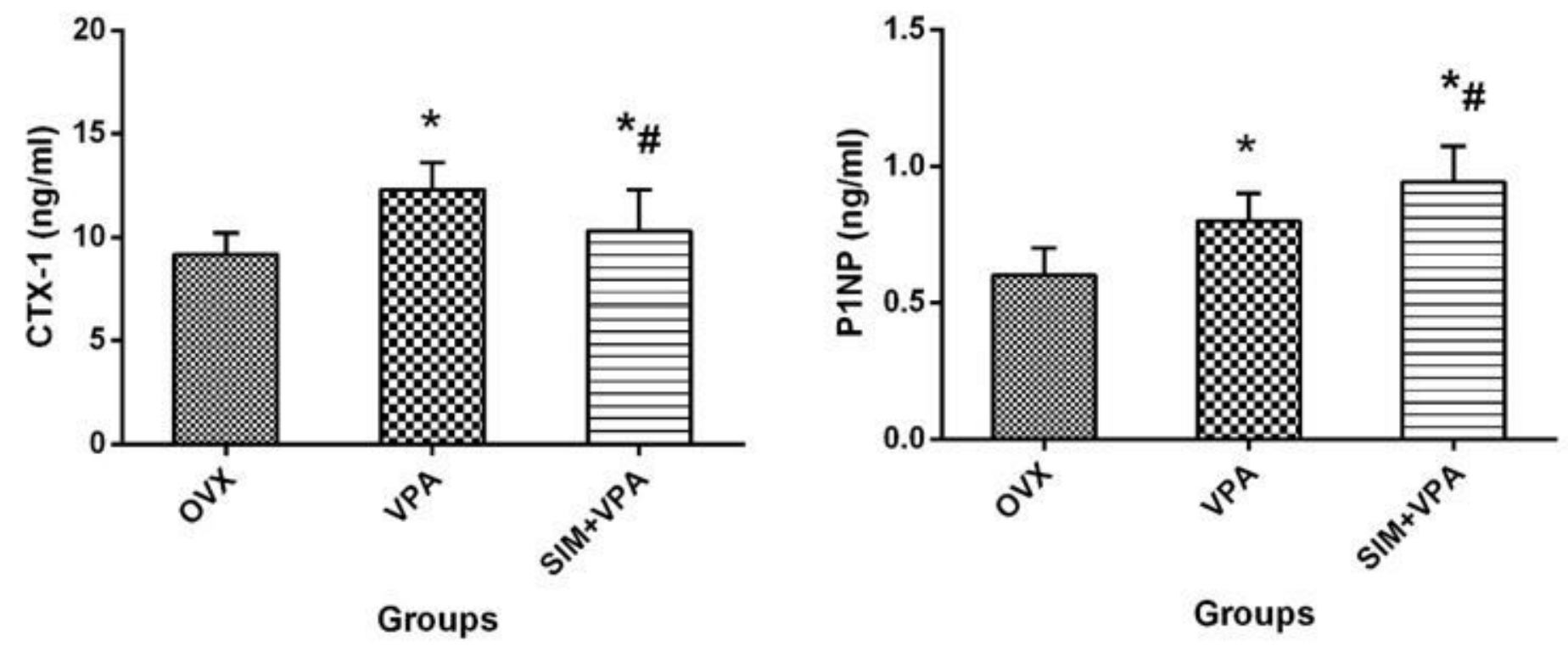

Figure 11

Bone turnover markers expressed as CTX-1 and P1NP for each group. ${ }^{*} p<0.05$ vs. OVX group, $\# p<0.05$ vs. VPA group. 